\title{
Efectos de estilos de liderazgo del director en la gestión de Instituciones Educativas
}

\section{Effects of the principal's leadership styles in the management of Educational Institutions}

DOI: $10.46932 / \mathrm{sfjdv} 2 \mathrm{n} 2-062$

Received in: january 1st, 2021

Accepted in: March 30th, 2021

\author{
Mg. Félix Eleazar De la Rosa Collao \\ Filiación: Universidad César Vallejo \\ Correo: felinodelarosa@hotmail.com \\ Dra. Isabel Menacho Vargas \\ Filiación: Universidad César Vallejo \\ Correo: isabelmenachov@gmail.com \\ Dra. María Ysabel Alvarez Huari \\ Filiación: Universidad Privada Norbert Wiener \\ Correo: alvarezhmariay@gmail.com
}

Mg. José Luis Camarena Mucha

Filiación: Universidad César Vallejo

Correo: arq.jlcamarena@gmail.com

\section{RESUMEN}

El problema de la gestión escolar es percibida como la deficiencia de la capacidad de los gestores educativos, en la cual el tipo de liderazgo no es claramente asumida por lo que los resultados en la escolaridad no alcancen las metas deseadas para el cambio sostenible del país, por ello, la importancia del estudio está en el fundamento que precisa la necesidad de generar conocimiento respecto a la forma de gestión, liderazgo así como del impacto en este nuevo proceso implementado a partir del año 2016, es un estudio de enfoque cuantitativo, básico de diseño no experimental con una muestra 116 docentes se utilizó la técnica de la encuesta y se aplicó instrumentos estandarizados con alta validez por juicio de expertos, los datos procesados en estadística descriptiva reportaron que el liderazgo es fuerte y la gestión educativa es buena, la prueba de hipótesis refrendo un Chi cuadrado de Wald 28,390 y una significancia de 0,000 menor a 0,05 determinando que el nivel de liderazgo y sus componentes inciden en los niveles de gestión de las instituciones educativas de la UGEL Parinacochas en la región Ayacucho, corroborando conclusiones de estudios previos.

Palabras clave: Liderazgo del director, Gestión Educativa.

\begin{abstract}
The problem of school management is perceived as the deficiency of the capacity of educational managers, in which the type of leadership is not clearly assumed so that the results in schooling do not reach the desired goals for sustainable change in the country. Therefore, the importance of the study is based on the need to generate knowledge regarding the form of management, leadership as well as the impact on this new process implemented since 2016, it is a study with a quantitative approach, basic of Non-experimental design with a sample of 116 participants, the survey technique was used and two standardized instruments with high validity by judges' criteria were applied, the data processed in
\end{abstract}


descriptive statistics reported that leadership is strong and educational management is good, the test of hypothesis endorsing a Chi square of Wald 28.390 and a significance of 0.000 less than 0.05 determining that the level of leadership and Its components affect the management levels of the educational institutions of the UGEL Parinacochas in the Ayacucho region, corroborating the conclusions of previous studies

Keywords: Principal's leadership, Educational Management.

\section{INTRODUCCIÓN}

Según el análisis (Furguerle y Vitorá, 2016) destaca la deficiencia de gestión y a la capacidad de liderazgo que se percibe en las instituciones públicas tales como las barreras comunicacionales, escasamente se reconoce e incentiva, resistencia de asumir políticas lo cual impide esfuerzos transformacionales, afectan directamente el aprendizaje de los estudiantes y en consecuencia a las deficiencias para situarse en los niveles de los estándares de formación de las competencias requeridas para su desarrollo personal e insertarse en el campo de la sociedad y el mundo laboral. (Iturra y Cancino, 2017) corrobora contar con marcos regulatorios tales como Marco de Buen Desempeño Directivo para fortalecer competencias del equipo directivo, constantes ajustes a las demandas y contextos, tipo de competencias y estándares de desempeño, construcción de comunidades de aprendizaje y desarrollo profesional

El análisis del estudio es importante dado que en la región Ayacucho, en la última década se observó deficiencias en los resultados de las mediciones de calidad educativa relacionado con los aprendizajes, el diagnostico regional determinó que una de las problemáticas esenciales fue el estilo de liderazgo que asumen los directivos (Fernández y Quintero 2017), dado que la tarea principal es la generación de altos niveles de logro de aprendizaje, en ese sentido es necesario identificar los factores que están incidiendo en la persistencia de la problemática, ya que desde el 2015, el proceso de meritocracia debió de haber impulsado la designación de directivos con el manejo de conocimientos, estrategias, que generen un clima adecuado y sobre todo, con alta capacidad de relaciones para tomar las decisiones oportunas en función al liderazgo pedagógico, (Hersey y Blanchard 2014) que se vinculen a los requerimientos de la sociedad, las cuales son la formación de competencias para insertarse a la dinámica del mundo laboral y el desarrollo profesional.

Asimismo, es pertinente analizar las innovaciones implementadas (Horn 2013) considerándose que las escuelas para dar un impulso de crecimiento y estar a la vanguardia de la ciencia, así como para las condiciones de alcanzar niveles de alta competitividad, requiere del reconocimiento base, diagnóstico de la organización, ya que de este modo el líder debe tomar la iniciativa en la sensibilización y comunicación de las metas propuestas (Montiel 2018) por ello, el diagnostico permite identificar la deficiencia del servicio educacional, por aspectos de gestión, de escasa decisión, así como de una 
convencional forma de seguir las pautas, en la cual se deja hacer sin guiar causa efectos negativos en el desarrollo formativo de los escolares (Prada, 2015) en consecuencia, el análisis de la alta trascendencia del liderazgo como eje central, sirve para revertir la realidad de los niveles de gestión, precisando la importancia de que el sistema educativo designe a gestores con alta capacidad de liderazgo.

Precisando que la gestión educativa y el liderazgo son temas de frecuente análisis (Maya y Aldana, 2019) las cuales derivan factores internos y externos que afectan a las organizaciones y que estos son de responsabilidad de las organizaciones y de la adaptación al sistema interactivo, (Montiel, 2012) por ello, el establecimiento de las relaciones son afectos determinantes en la consecución de metas previstas, así como del desarrollo institucional, (Rodríguez, 2011) asimismo, las investigaciones educativas, han demostrado que la preparación de los directivos, la tecnificación y sobre todo una maduración psicológica debe ser continua, para adaptarse a diversos contextos organizacionales, implicando confianza, respeto, tareas conjuntas haciendo que se genere un crecimiento organizacional, especialmente en instituciones de formación superior.

También se encuentra importancia en la determinación de las relaciones entre el liderazgo y la gestión (Weinstein, Muñoz, Sembler y Marfán, 2019), demostrando que toda organización está basada en la forma como se toman las decisiones, la organización de los recursos humanos bajo un principio armónico que influye positivamente en el clima, (Areche, 2013) los resultados indican que la confianza que se distribuye a cada uno de los integrantes implica alto nivel de compromiso e identidad, lo que hace que los integrantes alcancen mejores rendimientos en la gestión basada en competencias, (Bass y Burns, 2009) ya que el mejoramiento continuo son acciones que se traducen en la seguridad de la institución que presenta altos niveles de productividad.

En el campo educativo, el análisis del liderazgo es de importancia, ya que este concepto habiendo sido introducido en la gestión escolar, pareciera no tener el mismo efecto que en las empresas industriales y/o tecnológicas, dado que los resultados alcanzados no muestran una consolidación en la gestión de emprendimiento, en el desarrollo organizacional, menos aún en los productos que son representados por los niveles de aprendizaje de los estudiantes, por ello es necesario conocer los alcances de la competencia de gestión en la interacción con los demás, la toma de decisiones, así como el posicionamiento como una organización de calidad (Castro y Nader, 2014) ya que se ha determinado que el liderazgo es un conjunto de acciones que tiene la persona y se manifiesta al asumir retos, así como de alcanzar condiciones diversas para afrontar adversidades, haciendo que se interrelacionen las acciones individuales en acciones de conjunto dentro de una organización, (Covey, 2003) en consecuencia se considera que el líder influye en los demás, toma iniciativa, asume retos y es capaz de hacer que otros asuman los ideales, haciendo que se compenetren la necesidad, la capacidad, así como la consecuencia de la equidad y justicia. 
En el campo educativo, el liderazgo transformacional se ajusta a las condiciones de la organización escolar, dado que la visión de futuro, los retos y compromisos se adhieren en acciones de desarrollo a favor del aprendizaje (Evans, 2015; Hillca, 2015; y Horn 2013) estas características se sustentan en las bases axiológicas de las organizaciones, como el mantenimiento de la cultura institucional, se limita en los cambios o innovaciones, se permite mantener una misma hegemonía y no busca el cambio como medio, sino que se adapta a las circunstancias sin alejarse de su esencia, en las organizaciones productivas, (Maxwell, Raymond, Senge, Kotter, Archer, Santos y Davis, 2016) el estilo de liderazgo es sólido cuando se tiene un posicionamiento dentro del mundo económico, o cuando se tiene un espacio solido por los años de haber logrado el nivel de imagen sólido, (Monroy, 2013) en el contexto educativo, estos aspectos confluyen dentro de las características fundamentales del liderazgo pedagógico, en tal sentido las competencias parten de la articulación de los integrantes de la institución educativa, su extensión básica es el servicio al usuario que es el conocimiento de los estudiantes, con la cual la gestión se basa en la preparación acorde al nivel que se pretende lograr, para ello, se mantiene la misma esencia, así como se cuenta con recursos físicos y virtuales que deben ser adaptadas a la realidad.

Dentro de las acciones de los directores o responsables de la gestión escolar, están las relaciones que debe concretarse entre las organizaciones de la comunidad y la escuela, dado que los resultados deben ser el nivel de aprendizaje de los estudiantes, (Montiel, 2012; Párraga, y Bartolo, 2014) la integración y compromisos se basan en la preparación de ambientes saludables, uso de distintos recursos que permite el acrecentamiento de los conocimientos, así como de la experiencia que se obtiene en base a las interacciones entre el medio, la escuela y los agentes, (Prada, 2015) los directores de instituciones educativas, el conocimiento de administración es solo una parte de la gestión integral, a ello, debe articularse el conocimiento de la concreción de la currícula escolar, el proyecto de desarrollo comunal, así como del pleno dominio de las estrategias que deben aplicarse para alcanzar el desarrollo de las organizaciones.

En lo que respecta a la gestión de instituciones educativas, se encuentran dentro de los lineamientos de política educativa regido por el Ministerio de Educación, las mismas que son reguladas a través de las Resoluciones Ministeriales que norman los alcances de las metas para el año escolar en todas las instituciones del sistema educativo, (Montero, 2017) la gestión escolar trata el conjunto de acciones basadas en el cumplimiento de los compromisos de gestión especificadas en el marco del Buen Desempeño directivo, en la misma que se concreta dentro de los procedimientos de cumplimiento de las estrategias nacionales, regionales y locales que están concordantes con los instrumentos de gestión de cada institución educativa (Almadueña, 2018, Sotero 2017) la organización de los recursos humanos, la 
preparación de ambientes de aprendizaje, así como del fomento de las buenas practicas pedagógicas en consonancia con las disposiciones comunales que coadyuvan al buen desarrollo escolar.

Las concepciones de gestión (Chiavenato, 2009) precisa que toda organización requiere de un sistema de planeamiento estratégico, así como de un conjunto de personas como recursos indispensables convirtiéndose en el capital humano, (Steven y MacGuari 2017) las escuelas exitosas pasan por el fiel cumplimiento de los planes, con la capacidad de prevención y adelantamiento para minimizar riesgos, de este modo se afianza la productividad, que en el caso de las organizaciones escolares es el logro de aprendizaje y el posicionamiento en los altos niveles.

La gestión de instituciones educativas abarca las dimensiones de carácter pedagógica, social, comunal, administrativa o institucional, (Gonzales, 2015) dado que parte de una realidad con características particulares que buscan la generación de estructuras definidas por un marco normativo, las cuales se traducen en instrumentos de gestión para el manejo interno, sin embargo en todas ellas se encuentran precisadas las políticas de gobierno que deben aplicarse en el tiempo programado, (Cuenca, 2017, Minedu 2018, Maurtua 2019). En coherencia con lo planteado el objetivo del artículo es dar a conocer los aspectos que inciden en la percepción del liderazgo del director en la gestión de las instituciones educativas de la UGEL Parinacochas en la región Ayacucho, considerando los componentes basados en los estilos que asumen diversos gestores educativos, para comprender la problemática del estancamiento de la educación, así como exponer al conjunto de procedimientos empleados en el análisis de los fundamentos sobre el liderazgo y la gestión escolar.

\section{MÉTODO}

El presente artículo está concebido en enfoque cuantitativo describe el liderazgo del director y gestión institucional. Se apoyó en la metodología propuesta (Kerlinger y Lee 2002; Hernández, Fernández y Baptista 2018) sostienen la investigación descriptiva no experimental permite detallar situaciones y eventos. Es decir analiza la percepción del liderazgo del director en la gestión de las instituciones educativas (Abarca 2017; Rafelli 2019), asimismo, se precisa la síntesis de artículos de revisión nacionales e internacionales como base del análisis de la concepción del liderazgo y la gestión escolar.

La muestra estuvo conformado por 116 docentes de las instituciones educativas de la UGEL Parinacochas distrito de Coracora. Región Ayacucho.

La técnica para la recolección de datos se usó la encuesta adaptado del cuestionario Multifactorial Estandarizado de Liderazgo (MLQ Forma 5xcorta) de Bass y Avolio, se sometieron a juicio de expertos y validados a través de la formula Crombach con datos de la muestra piloto. Está conformado de 118 items 
La información recolectada se trasladó a una hoja Excel y seguidamente el procesamiento estadístico de los datos para resolver y probar las hipótesis planteadas (Kerlinger y Lee, 2002; Hernández, Fernández y Baptista 2018) para ello se da cuenta del uso de la tecnología, dado que los datos fueron procesados mediante el software estadístico SPSS22.0.

A razón de la presentación de los hallazgos a nivel descriptivo e inferencial, se utilizó el método inductivo, triangulación teórica para contrastar y/o discutir asumiendo una postura propia que permita identificar características particulares y de ello poder generalizar a razón de la magnitud de los resultados, ya que la muestra siendo probabilística abarco una población amplia (Abarca 2017; Rafelli 2019), del mismo modo, en base a los procedimientos del método hipotético deductivo se presenta la concordancia y diferencia halladas a nivel teórico y de estudios empíricos (Kerlinger y Lee 2002; Hernández, Fernández y Baptista 2018), por lo que las conclusiones permiten el incremento de las bases teóricas de cada uno de los factores, dimensiones, y variables estudiadas.

\section{RESULTADOS}

El reporte del análisis estadístico realizó mediante la herramienta SPSS 22.1, para las variables y sus componentes, dado las características de niveles se expone en una figura de frecuencias; en la cual una mayoría del $43.9 \%$ asigno el nivel de un Liderazgo Medio y una minoría indico un nivel de liderazgo alto mientras que un $18.1 \%$ califico como de nivel bajo, observándose la tendencia que predomina es positivo de nivel media a nivel alta.

Figura: distribución del nivel de Liderazgo del director y sus dimensiones

\section{Liderazgo del director y sus dimensiones}

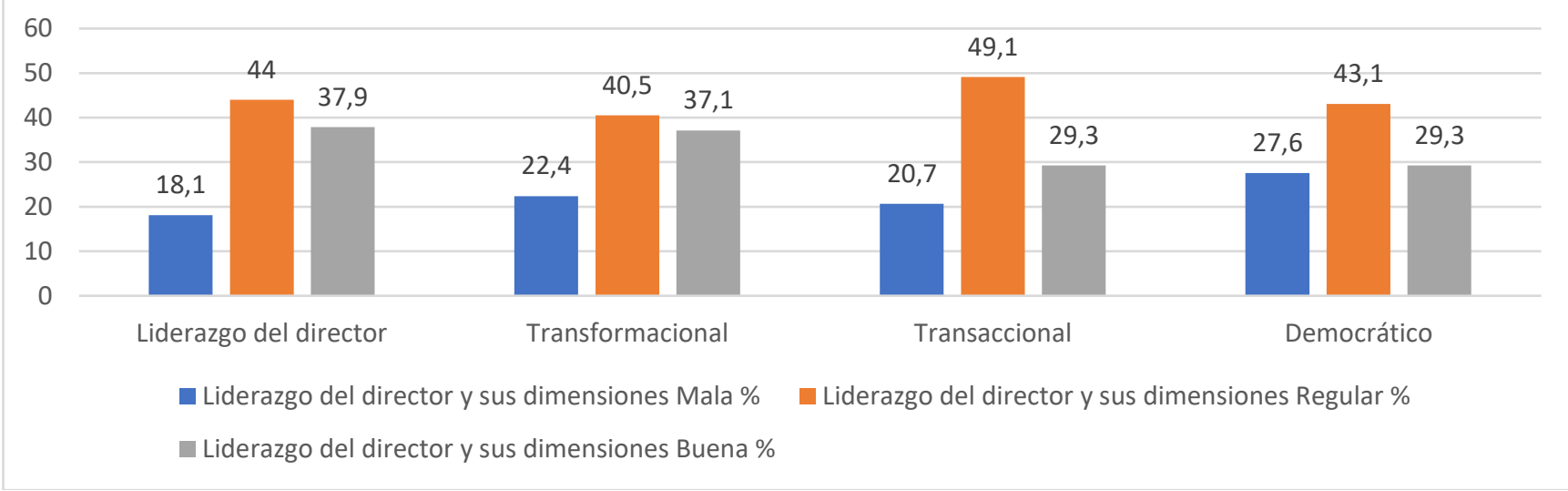

Respecto a las dimensiones analizadas el reporte indica que una mayoría compuesta por el $47.4 \%$ asignó el nivel regular de la gestión, mientras que para una minoría compuesto por 37.9\% de la muestra 


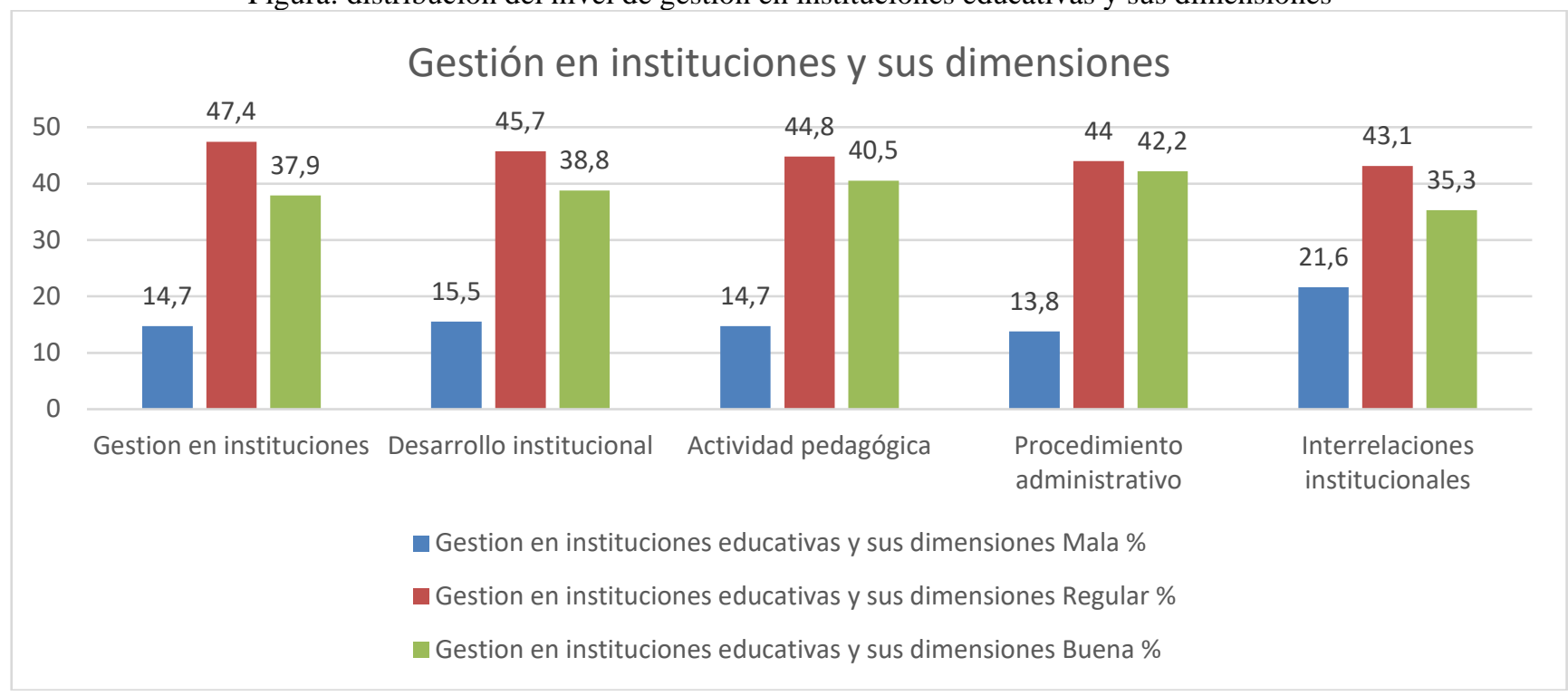

Los resultados de la prueba de hipótesis determinaron que el liderazgo del director tiene incidencia directa y significativa en la gestión educativa de los docentes de las instituciones educativas de la UGEL Parinacochas - Ayacucho, a razón del análisis del método de regresión ordinal con un Chi cuadrado = 162,930 y un nivel de significancia $=0,000$, así como de la prueba de medidas equivalentes de seudo cuadrado de los modelos lineales partiendo del factor predictivo del liderazgo del director frente a la dependencia de la gestión de instituciones educativas se halló una variabilidad de $86.2 \%$ determinando una incidencia de la población analizada.

Estimación de los parámetros de la Gestión educativa en el liderazgo del director

\begin{tabular}{|c|c|c|c|c|c|c|c|c|}
\hline & & \multirow[t]{2}{*}{ Estimación } & \multirow[t]{2}{*}{$\begin{array}{l}\text { Error } \\
\text { estándar }\end{array}$} & \multirow[t]{2}{*}{ Wald } & \multirow[t]{2}{*}{$\mathrm{gl}$} & \multirow[t]{2}{*}{ Sig. } & \multirow{2}{*}{$\begin{array}{l}95 \% \text { de } \\
\text { confianza }\end{array}$} & \multirow{2}{*}{$\begin{array}{l}\text { intervalo } \\
\text { Límite } \\
\text { superior } \\
\end{array}$} \\
\hline & & & & & & & & \\
\hline \multirow[t]{2}{*}{ Umbral } & {$[$ Gestioneducativa $=1]$} & $-9,533$ & 1,247 & 58,471 & 1 & ,000 & $-11,976$ & $-7,089$ \\
\hline & [Gestioneducativa = 2] & $-3,045$ &, 724 & 17,695 & 1 & 000 & $-4,463$ & $-1,626$ \\
\hline \multirow[t]{3}{*}{ Ubicación } & [competenciadeldirect $=1$ ] & $-10,855$ & 1,356 & 64,051 & 1 & 000 & $-13,514$ & $-8,197$ \\
\hline & [competenciadeldirect $=2]$ & $-6,289$ & 1,031 & 37,193 & 1 & 000 & $-8,310$ & $-4,268$ \\
\hline & [competenciadeldirect=3] & $0^{\mathrm{a}}$ & , & & 0 & & . & . \\
\hline
\end{tabular}

Función de enlace: Logit.

a. Este parámetro está establecido en cero porque es redundante.

En conclusión, el análisis de regresión logística ordinal indicó un puntaje Chi cuadrado de Wald 37,193 mayor a 4 puntos, y el valor $\mathrm{p}=0,000<\alpha 0,01$ lo cual significó que a medida que el liderazgo del 
director mejora, también mejora la gestión educativa de manera significativa la misma que fue percibida por los docentes de las instituciones educativas de la UGEL Parinacochas - Ayacucho.

\section{DISCUSIÓN}

Del reporte estadístico, los resultados significan que si bien es cierto la tendencia del liderazgo y de la gestión están mejorando paulatinamente, no basta para alcanzar el desarrollo institucional y organizacional, por lo que se debe impulsar programas de mejoramiento, de sustentabilidad mediante acciones guiadas para elevar el nivel de calidad educativa, además, el análisis determinó las incidencias de la gestión de los directores en las instituciones educativas deben estar basadas en el liderazgo, ya que son responsables de las posibles causas de una inadecuada gestión, así como de una baja de la realidad lo que podría estar incidiendo de manera negativa en el nivel de aprendizaje y logro de capacidades y competencias de los estudiantes.

Por ello, en concordancia con las investigaciones nacionales e internacionales se resalta el proceso formativo del nivel de liderazgo, es decir que para una gestión establecen que los educadores deben especializarse en conocimientos de la gestión organizacional, por ello, es que en la realidad se encuentra solo un nivel medio de liderazgo percibido en los directores (43.9\%) precisando que las capacidades de concertación, comunicación trabajo en equipo no están consolidadas, (Caloway 2015; Chiavenato 2009; Covey 2003), sin embargo se aprecia una tendencia favorable al desarrollo y crecimiento de la misma; del mismo modo el análisis de la percepción de la gestión de instituciones educativas, también fueron determinadas como de nivel regular, es decir que ambas variables estadísticamente se sitúan en los niveles y/o rangos intermedios, sin embargo la tendencia es positiva.

Otro de los aspectos coincidentes en las investigaciones es la determinación que la competencia del liderazgo aun no es clara y desarrollada en los gerentes educativos, (Areche 2013; Bertha 2016; Castro y Nader 2014) dado que la gestión escolar en el sistema educativo peruano se encuentra dentro de los niveles de crecimiento, siendo preponderante el nivel intermedio, lo que indica la urgencia de reformular el sistema de mejoramiento de la gestión escolar.

Sobre el procedimiento metodológico para la prueba de hipótesis en estudios de ciencias sociales en educación se ajusta al modelo de regresión ordinal, por lo que se determinó que el liderazgo del director incide de manera significativa en la gestión educativa (Hernández et al 2010; Amón 2002; Kerlinger y Lee 2002; MacGuari 2019), así como se resalta que el liderazgo está relacionado de manera directa con la gestión de directores en organizaciones educativas y estas se encuentran en etapa de maduración (Maurtua 2019; Prada 2015; Sotero 2017; Ralph 2016; Bass y Avolio 1990) este proceso debe alcanzarse en procesos de interacción, capacitación ya que la capacidad de liderazgo de los gerentes de organizaciones, determinan el nivel de producción y crecimiento posesionándose en los diversos 
contextos y mercados, estableciendo que la función básica del líder es integrar ideas y coadyuvar al logro de metas comunes, (Montiel 2018; Gonzales 2015; Montero 2017; Maya y Aldana 2019) estos aspectos son plasmados en los lineamientos de política educativa, por ello la importancia de los lideres dentro de las organizaciones educativas basado en liderazgo general de alta competencia y posicionamiento de la organización escolar, (Montero 2017; Minedu 2017) que deriva el proceso gradual del cambio de la institución mediante la aplicación sistemática de mejora pasa por la asimilación de renovación de comprensión de las nuevas necesidades ante ello el liderazgo es esencial para el cumplimiento de los procesos educativos.

Otra especificación es que los fundamentos teóricos y prácticos no han evidenciado estudios aplicados directamente en la realidad educativa, sino que los factores de gestión solo se han adaptado de los postulados de las organizaciones industriales, tecnológica, por lo que es una tarea de la ciencia establecer principios y leyes que faculten una consistente base de formación de líderes educativos, básicamente en las instituciones educativas rurales con realidad diversa y solo se refrenda con percepciones que establecen el nivel regular que es una tendencia.

\section{CONCLUSIONES}

Primero: el estilo de liderazgo de director y la gestión de la institución educativa se relaciona con capacidad de liderazgo, alta preparación, madurez psicológica, adaptación y crecimiento a diversos contextos organizacionales, en clima positivo, basado en competencia y crecimiento continuo

Segundo : en reporte estadístico las tendencias de percepción docente en el nivel de los estilos liderazgo del director, transformacional, transaccional y democrático la mayoría $44 \%$ asigno el nivel de liderazgo regular, mientras $37.9 \%$ nivel buena y $18.1 \%$ nivel mala de las instituciones educativas de la UGEL Parinacochas de la región Ayacucho

Tercero: en cuanto a las gestiones destacan 47,4\% nivel regular, 37,9\% califica nivel buena y 14,7\% nivel mala. Encontrándose tendencia positiva en gestión educativa de las instituciones educativas de la UGEL Parinacochas de la región Ayacucho

Cuarto: el análisis de regresión logística ordinal Chi cuadrado de Wald 28,390 y una signicancia de 0,000 menor a 0,05 determina a mayor liderazgo directivo mejora la gestión educativa de las instituciones educativas de la UGEL Parinacochas 


\section{REFERENCIAS}

Aliaga, J. (2014). Competencia directivo y el desempeño docente en Instituciones Educativas Públicas del nivel Primaria en la RED $N^{\circ}$ 04, UGEL $N^{\circ}$ 02; Independencia, 2017. Lima - Perú: (Tesis de maestría) Universidad César Vallejo.

Areche, T. (2013). La gestión institucional y calidad en el servicio educativo según la percepción de docentes y padres de familia del $3^{\circ}, 4^{\circ}$ y $5^{\circ}$ de secundaria del colegio María Auxiliadora de HuamangaAyacucho, 2011. Tesis para optar el grado de licenciado en educación. Lima, Perú: Universidad Nacional Mayor de San Marcos.

Almadueña, M. (2018). La Competencia de Gestión en educación. Madrid: La Muralla.

Amon, J. (2002). Estadística para investigaciones en psicología. Madrid: Narcea

Bass T y Avolio F. (1990). Adaptación del cuestionario Multifactorial. México: Mc Graw Hill.

Bass T y Burns P. (2009). Teoria del Liderazgo transformacional. Mexico: Mac Graw Hill.

Caloway, H. (2015). Teoría y diseño organizacional. México DF: Prentice Hall.

Castro, A., y Nader, M. (2014). Estilos de liderazgo, contexto y cultura organizacional un estudio comparativo en población civil y militar. Buenos Aires: Paidós.

Chiavenato, I. (2009). Gestión del talento humano. México: McGraw Hill.

Covey, S. (2003). Liderazgo centrado en principios. Barcelona: Paidós Ibérica.

Cuenca, R. (2017). La Competencia en las Escuelas: Una vision del desarrollo en el aprendizaje. Madrid: Narcea.

Evans R. (2015). Interacción entre inteligencia emocional y estilos de liderazgo en directivos de instituciones educativa: Caso Peruano. España: Universidad de Valencia.

Fernandez M y Quintero N. (2017). Liderazgo transformacional y transaccional en emprendedores venezolanos. Revista Venezolana de Gerencia, doi:https://www.redalyc.org/articulo.oa?id=2905145700

Furguerle R y Vitolá M. (2016). Liderazgo en los directivos de educacion primaria. Redalyc.org, Vol 18 №2, 1-21. doi:https://www.redalyc.org/articulo.oa?id=99345727004

Gonzales, N. (2015). Competencia del conocimiento, competencias, competencia e impacto en la Gestión educativa del centro. Estudio de un caso de un CRA. Zaragoza - España: Universidad de Zaragoza Hernandez, R., Fernandez, C., y Baptista. (2016). Metodologia de la investigación. México: Sexta Edición Mac Graw Hill Education.

Hersey, G., y Blanchard, F. (2014). Liderazgo situacional. México: Ediciones Pearson.

Hillca, T. (2015). Liderazgo transformacional y desempeño docente en la especialidad de Ciencias Históricos Sociales del Instituto Pedagógico Nacional Monterrico. Tesis para optar el grado de magister 
en educación. Lima: Universidad Mayor de San Marcos.

Horn, K. (2013). Liderazgo escolar en Chile y su influencia en los resultados de aprendizaje. Tesis para optar el grado de licenciado en educación. Madrid: universidad autónoma de Madrid.

Iturra C y Cancino V. (2017). Asesoramiento a equipos de gestión y liderazgo educativo desde el abordaje de las competencias funcionales. Scielo, Vol $26 \mathrm{~N}^{\circ}$ 101. doi:https://doi.org/10.1590/s010440362018002601381

Kerlinger, F., y Lee, H. (2002). Investigación del comportamiento. México: Pearson educación.

Maya, E., y Aldana, J. (2019). Liderazgo Directivo y Educación de Calidad. Año V Vol N ${ }^{\circ}$ 9. doi:ISSNL: 2542-3029; ISSN: 2610-802X

Maurtua, K. (2019). Gerencia Avanzada en Educación Liderazgo directivo como elemento estratégico en la efectividad del desempeño docente. Tesis para optar el grado de magister en educación. Bárbula, Venezuela: Universidad de Carabobo.

MacGuari, L. (2017). Habilidades directivas. Madrid: Thomsom

MINEDU. (2017). Lineamientos para la Competencia escolar. Lima: Documento de trabajo sin editar.

Montero, C. (2017). La direccción de las Escuelas que queremos. Lima Perú: Mantaro.

Monroy, J. (2013). Educación Liderazgo directivo como elemento estratégico en la efectividad del desempeño docente. Tesis para optar el grado de magister en educación. Venezuela: Universidad de Carabobo

Montiel, C. (2018). El liderazgo transformacional del directivo y el desempeño laboral de los docentes en las instituciones educativas de nivel primario. Tesis para optar el grado de maestro en educación. Lima: Universidad San Ignacio de Loyola

Párraga, T. y Bartolo, R. (2014). Liderazgo transformacional del director y el desempeño del trabajador de servicio en las Instituciones Educativas según los docentes de la Red 18-Ate, 2014. Tesis para optar el grado de licenciada en educación. Madre de Dios: Universidad Nacional Amazónica De Madre De Dios.

Prada, L. (2015). El estilo de liderazgo del director y el desempeño docente en la calidad educativa, en las instituciones educativas públicas de la capital de la provincia de Canta de la Ugel $N^{\circ} 12$, región Lima provincias. Lima - Perú: Universidad Nacional de Educación Enrique Guzmán y Valle.

Ralph, S. (2016). Teorías del liderazgo - Teoría de los rasgos “El líder nace, no se hace”. México D.F.: Trillas.

Rodriguez, G. (2011). Funciones y rasgos del liderazgo en los centros de enseñanza Educación y $\begin{array}{lllll}\text { Educadores. } & \text { (U. } & \text { d. } & \text { Sabana, } & \text { Ed.) }\end{array}$ doi:https://www.redalyc.org/articulo.oa?id=83421404003

Sotero, M. (2017). Gestión pedagógica del trabajo docente a través de grupos corporativos. Tesis para optar el grado de magister en educación. Lima-Perú: Pontificia Universidad Católica del Perú. 
Tuesta, L. (2018). Funciones del directivo de las escuelas Bolivarianas. Caracas - Venezuela: (Tesis de maestria) Universidad Rafael Marial Barnal

Vadillo, M. (2016). Liderazgo y motivación de equipos de trabajo. Madrid: Editorial ESIC.

Weinstein, J., Muñoz, G., Semble,r M., y Marfán, J. (2019). Un década de investigación empirica sobre el liderazgo educativo en Chile. Un Revisión sistematica de los articulos publicados en revista indexadas (2008-2019). Calidad en la Educación, 15-52 